\title{
A note on eigenvalues of ordinary differential operators
}

Alan Ho

In this follow-up on the work of [FS] an improved condition for the discrete eigenvalues of the operator $-d^{2} / d x^{2}+V(x)$ is established for $V(x)$ satisfying certain hypotheses. The eigenvalue condition in [FS] establishes eigenvalues of this operator to within a small error. Through an observation due to C. Fefferman, the order of accuracy can be improved if a certain condition is true. This paper improves on the result obtained in [FS] by showing that this condition does indeed hold.

The theorem proven here relies on a version of WKB theory developed in [FS] and applies to operators with large slowly varying potentials. For example, it applies to potentials of the form $V(x)=\lambda^{2} V_{1}(x)$ for fixed, smooth $V_{1}$, with $V^{\prime \prime}>0, V$ having a local minimum, and $\lambda \gg 1$. The theorem applies to more general potentials as well.

Standard WKB theory yields the statement that all eigenvalues $E$ of the differential operator $-d^{2} / d x^{2}+V(x)$ satisfy

(1) $\int_{x_{\text {left }}}^{x_{\text {right }}}(E-V(x))^{1 / 2} d x=\pi\left(k+\frac{1}{2}\right)+O\left(\lambda^{-1}\right), \quad$ for some $k \in \mathbb{Z}$,

where $x_{\text {left }}$ and $x_{\text {right }}$ are the two solutions of $E-V(x)=0$.

[FS] shows that this condition for eigenvalues can be improved so that given $N>0$, there exists $N^{\prime}>0$ and complex functions $h_{l}(E)$ defined in [FS] so that (1) becomes

$$
\int_{x_{\mathrm{left}}}^{x_{\mathrm{right}}}(E-V(x))^{1 / 2} d x+\operatorname{Im} \log \left(1+\sum_{l=1}^{N^{\prime}} h_{l}(E)\right)
$$




$$
=\pi\left(k+\frac{1}{2}\right)+O\left(\Lambda^{-N}\right),
$$

where $\Lambda$, which will be defined precisely in the theorem, plays a role analogous to $\lambda$. $h_{1}$ is explicitly given in [FS] and is purely imaginary. For the moment however, the critical property of $h_{l}$ is that $h_{l}(E)=$ $O\left(\Lambda^{-l}\right)$, and the quantity $\sum h_{l}(E)$ is $O\left(\Lambda^{-1}\right)$ in absolute value, and hence the Taylor series of $\log$ gives

$$
\int_{x_{\mathrm{left}}}^{x_{\mathrm{right}}}(E-V(x))^{1 / 2} d x+i h_{1}(E)=\pi\left(k+\frac{1}{2}\right)+O\left(\Lambda^{-2}\right) .
$$

But if we were to carry out the same calculation to order $\Lambda^{-3}$, then since

$$
\log \left(1+\sum_{l=1}^{N^{\prime}} h_{l}(E)\right)=h_{1}(E)+h_{2}(E)-\frac{1}{2} h_{1}^{2}(E)+O\left(\Lambda^{-3}\right)
$$

we have

$$
\begin{aligned}
& \int_{x_{\text {left }}}^{x_{\text {right }}}(E-V(x))^{1 / 2} d x+ \operatorname{Im}\left(h_{1}(E)+h_{2}(E)\right) \\
&=\pi\left(k+\frac{1}{2}\right)+O\left(\Lambda^{-3}\right) .
\end{aligned}
$$

Note $h_{1}^{2}$ is real and therefore makes no contribution to the left-hand side of (5). Moreover, we shall show that $h_{k}$ is purely imaginary whenever $k$ is odd and real whenever $k$ is even. This reduces the left-hand side of (5) to the simpler left-hand side of (3). This improves upon (3) since (5) holds to $O\left(\Lambda^{-3}\right)$ instead of $O\left(\Lambda^{-2}\right)$. Using the above fact we obtain an improved version of part of the WKB Eigenvalue Theorem. (cf. [FS, p. 239]). For the reader's convenience and for completeness we repeat the hypotheses here.

Theorem. Suppose we are given positive functions $S(x)$ and $B(x)$ on $I$ and a potential $V(x)$ supported on a possibly unbounded interval $I_{\mathrm{BVP}}$ with $I \subset I_{\mathrm{BVP}}$. Furthermore, suppose we are given two real numbers $E_{0} \leq E_{\infty}$, positive numbers $\varepsilon<1 / 100, K>1$ and $N>K \varepsilon^{-10}$. Define $N^{\prime}=[\varepsilon N / 500]$ and $N^{\prime \prime}=3 \varepsilon N^{\prime} / 2-K-33$. And suppose we have the following hypotheses: 
Hyp0) If $x, y \in I$ and $|x-y|<c B(x)$, then

$$
c<\frac{B(y)}{B(x)}<C \quad \text { and } \quad c<\frac{S(y)}{S(x)}<C .
$$

Hyp1) For $x \in I$ and $\alpha \geq 0$ we have

$$
\left|\left(\frac{d}{d x}\right)^{\alpha} V(x)\right| \leq C_{\alpha} S(x) B^{\alpha}(x) .
$$

Hyp2) The equation $V(x)=E_{0}$ has two solutions $x_{\text {left }}<x_{\text {right }}$ in $I$, and they satisfy

$$
\operatorname{dist}\left(x_{\text {left }}, \partial I\right)>c B\left(x_{\text {left }}\right), \quad \operatorname{dist}\left(x_{\text {right }}, \partial I\right)>c B\left(x_{\text {right }}\right) .
$$

Hyp3)

$$
-V^{\prime}(x)>c S\left(x_{\mathrm{left}}\right) B^{-1}\left(x_{\mathrm{left}}\right), \quad \text { for } x \in\left[x_{\mathrm{left}}, x_{\mathrm{left}}+c_{1} B\left(x_{\mathrm{left}}\right)\right]
$$

and

$V^{\prime}(x)>c S\left(x_{\text {right }}\right) B^{-1}\left(x_{\text {right }}\right), \quad$ for $x \in\left[x_{\text {right }}-c_{1} B\left(x_{\text {right }}\right), x_{\text {right }}\right]$.

Нyp4)

$$
c S(x)<E_{0}-V(x)<C S(x)
$$

for $x \in\left[x_{\text {left }}+c_{1} B\left(x_{\text {left }}\right), x_{\text {right }}-c_{1} B\left(x_{\text {right }}\right)\right]$.

To state the remaining hypotheses, it is convenient to establish some notation. Set $\lambda(x)=S^{1 / 2}(x) B(x)$ for $x \in I$, and set

$$
\begin{aligned}
& B_{\text {left }}=B\left(x_{\text {left }}\right), \quad S_{\text {left }}=S\left(x_{\text {left }}\right), \quad \lambda_{\text {left }}=\lambda\left(x_{\text {left }}\right) . \\
& B_{\text {right }}=B\left(x_{\text {right }}\right), \quad S_{\text {right }}=S\left(x_{\text {right }}\right), \quad \lambda_{\text {right }}=\lambda\left(x_{\text {right }}\right) .
\end{aligned}
$$

For $\left|E-E_{0}\right|<c S_{\text {left }}$, let $x_{\text {left }}(E)$ be the solution of $V(x)=E$ nearest to $x_{\text {left }}$, and for $\left|E-E_{0}\right|<c S_{\text {right }}$, let $x_{\text {right }}(E)$ be the solution of $V(x)=E$ nearest to $x_{\text {right }}$. Define

$$
S_{\text {min }}=\int_{x_{\text {left }<x<x_{\text {right }}}} S(x) d x
$$


406 A. Ho

and

$$
\Lambda=\int_{x_{\text {left }}}^{x_{\mathrm{right}}}\left(S^{1 / 2}(x) B^{2}(x)\right)^{-1} d x .
$$

Our remaining hypotheses are as follows.

- Assumptions on $V(x)$ in all of $I_{\mathrm{BVP}}$ :

Hyp5) If $\left|E-E_{0}\right|<c_{2} S_{\min }$ and $E \leq E_{\infty}$, then $V(x)>E$ for all $x \in I_{\mathrm{BVP}}-\left[x_{\text {left }}(E), x_{\text {right }}(E)\right]$.

Hyp6) If $x \in I_{\mathrm{BVP}}$ satisfies $x<x_{\text {left }}-\lambda_{\text {left }}^{K} B_{\text {left }} / 2$ then $V(x) \geq$ $E_{\infty}+100 /\left|x-x_{\text {left }}\right|^{2}$, and if $x \in I_{\mathrm{BVP}}$ satisfies $x>x_{\text {right }}+\lambda_{\text {right }}^{K} B_{\text {right }} / 2$, then $V(x) \geq E_{\infty}+100 /\left|x-x_{\text {right }}\right|^{2}$.

- Technical Assumptions:

Hyp7) $\max _{x \in I} S(x) \leq \lambda_{\text {left }}^{K} S_{\text {left }}$ and $\max _{x \in I} S(x) \leq \lambda_{\text {right }}^{K} S_{\text {right }} \cdot$

Hyp8)

$$
\int_{x_{\text {left }}}^{x_{\text {right }}}\left(\frac{d x}{S^{1 / 2}(x)}\right) \leq \Lambda^{K} \min \left\{S_{\text {left }}^{-1 / 2} B_{\text {left }}, S_{\text {right }}^{-1 / 2} B_{\text {right }}\right\} .
$$

Нyp9)

$$
\left(\int_{x_{\text {left }}}^{x_{\text {right }}} \frac{d x}{S^{1 / 2}(x) B^{4}(x)}\right)\left(\int_{x_{\text {left }}}^{x_{\text {right }}} \frac{d x}{S^{1 / 2}(x)}\right) \leq \Lambda^{K} .
$$

\section{- WKB Condition:}

Hyp10) $\Lambda$ is bounded below by a positive constant depending only on $\varepsilon, K$ and $N$, and on $c, C, c_{1}, c_{2}, C_{\alpha}$ in Hyp0)-Нyp4).

Then if $E$ is an eigenvalue of $-d^{2} / d x^{2}+V(x)$, we have that

$$
\int_{x_{\mathrm{left}}}^{x_{\mathrm{right}}}(E-V(x))^{1 / 2} d x+i h_{1}(E)=\pi\left(k+\frac{1}{2}\right)+\phi_{\mathrm{error}}(E),
$$

with $\left|\phi_{\text {error }}\right| \leq C \Lambda^{-3}$ and

$$
h_{1}(E)=\frac{i}{48} \lim _{\delta \rightarrow 0}\left(\int_{x_{\mathrm{left}}+\delta}^{x_{\mathrm{right}}-\delta} V^{\prime \prime}(x)(E-V(x))^{-(3 / 2)} d x-q(E) \delta^{-1 / 2}\right)
$$

with $q(E)$ uniquely specified by demanding the finiteness of the limit. 
ProOF. Let us say that a complex function $f_{l}$ has the alternating parity property on the index $l$ if it is real-valued for $l$ even and purely imaginary for $l$ odd. It suffices to show that $h_{l}$ has the alternating parity property on the index $l$. Recall that the $h_{l}$ 's are inductively determined by

$$
u_{k}^{\text {left }}(x, E)=\sum_{l=0}^{k} h_{l}(E) u_{k-l}^{\text {right }}(x, E),
$$

where $u_{k}$ is the canonical solution of the transport equations

$$
\begin{gathered}
u_{0} \equiv 1, \\
2 i u_{k+1}^{\prime}+\left(\frac{5}{16}\left(p^{\prime}\right)^{2} p^{-5 / 2}-\frac{1}{4} p^{\prime \prime} p^{-3 / 2}\right) u_{k} \\
-\frac{1}{2} p^{\prime} p^{-3 / 2} u_{k}^{\prime}+p^{-1 / 2} u_{k}^{\prime \prime}=0, \quad 0 \leq k<N^{\prime} .
\end{gathered}
$$

In particular, since $u_{0}^{\text {left }}=u_{0}^{\text {right }}=1$,

$$
h_{2}(E)=u_{2}^{\text {left }}(x, E)-h_{1}(E) u_{1}^{\text {right }}(x, E) .
$$

Since $h_{1}$ is known to be purely imaginary, it suffices to show $u_{k}^{\text {left }}$ and $u_{k}^{\text {right }}$ each have the alternating parity property on the index $k$. Let us show $u_{k}^{\text {left }}$ has the alternating parity property; the proof for $u_{k}^{\text {right }}$ is totally analogous.

Lemma 10 of $[\mathrm{FS}]$ relate the canonical solution to the elementary solution of the transport equations in the following manner: if $u=\left(u_{0}(x), u_{1}(x), \ldots, u_{N^{\prime \prime}}(x)\right)$ is the canonical solution of the transport equations, and if $\tilde{u}=\left(\tilde{u}_{0}, \ldots, \tilde{u}_{N^{\prime \prime}}(x)\right)$ is the elementary solution, then

$$
u_{k}(x)=\sum_{l=0}^{k} w_{k-l, 0} \tilde{u}_{l}(x),
$$

where $w_{k l}$ will be investigated in more detail below. Since the construction of the elementary solutions in [FS] makes it clear $\tilde{u}_{l}$ has the alternating parity property on the index $l$, we have reduced the problem to showing $w_{k l}$ has the alternating parity property on the index $k$. Equivalently, letting $w_{k}(x)=\sum_{-3 k<l} w_{k l} x^{l / 2}$, it suffices to show $w_{k}$ has the alternating parity property on the index $k$.

Now all that is needed is to take account of the real and purely imaginary quantities that arise in the construction of $w_{k}$. (cf. [FS, 
p. 155-162, 171]). We proceed as follows: $w_{k}(x)$ can be written in terms of $h_{k l}^{\#}, q_{k l}^{\#}$ and $\hat{h}_{k l}$ via the equation

$$
\begin{aligned}
\left(1+\sum_{k=1}^{N} \lambda^{-k} w_{k}(x)\right)=( & \left(1+\sum_{k=1}^{2 N} \sum_{l=2-k}^{3 N} h_{k l}^{\#} x^{l / 2} \lambda^{-k}+O\left(\lambda^{-\varepsilon N / 4}\right)\right. \\
& \cdot\left(1+\sum_{k=1}^{N} \sum_{l=-k}^{N} q_{k l}^{\#} x^{l} \lambda^{-2 k}+O\left(\lambda^{-\varepsilon N / 4}\right)\right) \\
& \left.\cdot\left(1+\sum_{k=1}^{N} \sum_{l=-3 k}^{N} \hat{h}_{k l} x^{l / 2} \lambda^{-k}+O\left(\lambda^{-\varepsilon N / 4}\right)\right)\right) .
\end{aligned}
$$

To prove $w_{k}$ has the alternating parity property on the index $k$, we will want to show both $h_{k l}^{\#}$ and $\hat{h}_{k l}$ have this property on the index $k$ and $q_{k l}^{\#}$ is real. Let us first look at $h_{k l}^{\#}$. [FS] shows

$$
\begin{aligned}
\exp \left(\sum_{k=1}^{N} \sum_{l=-k}^{N} h_{k l} x^{l+3 / 2} \lambda^{-(2 k-1)}\right) & \\
= & \left(1+\sum_{k=1}^{2 N} \sum_{l=2-k}^{3 N} h_{k l}^{\#} x^{l / 2} \lambda^{-k}+O\left(\lambda^{-\varepsilon N / 4}\right)\right),
\end{aligned}
$$

where the right-hand side is a high-order Taylor expansion with remainder. Let us consider more carefully how $h_{k l}^{\#}$ depends on $h_{k l}$. Note that

$$
\begin{aligned}
\frac{2 i}{3} \lambda\left(y_{0}(x)\right)^{3 / 2} & \sum_{k=1}^{N} \sum_{l=-k}^{N} f_{k l}^{\# \#} x^{l} \lambda^{-2 k} \\
& =\sum_{k=1}^{N} \sum_{l=-k}^{N} h_{k l} x^{l+3 / 2} \lambda^{-(2 k-1)}+O\left(\lambda^{-\varepsilon N / 4}\right) .
\end{aligned}
$$

Since $y_{0}(x)$ and $f_{k l}^{\# \#}$ are real, $h_{k l}$ is purely imaginary since it depends only on these quantities multiplied by $i$. Now set

$$
X=\sum_{k=1}^{N} \sum_{l=-k}^{N} h_{k l} x^{l+3 / 2} \lambda^{-(2 k-1)}
$$

A sufficiently high power of $X$ will be $O\left(\lambda^{-\varepsilon N / 4}\right)$, so the left-hand side of (8) has a Taylor expansion with remainder. Note that $X^{s}$ is purely 
imaginary if and only if $s$ is odd. Since $X$ contains nothing but odd powers of $\lambda$, one finds upon collecting terms of the Taylor expansion with respect to $\lambda$ that the coefficients are purely imaginary for all odd powers of $\lambda$, real for all even powers of $\lambda$. This says precisely that $h_{k l}^{\#}$ has the alternating parity property on the index $k$.

Now let us consider $q_{k l}^{\#}$. Quite simply, $q_{k l}^{\#}$ is real since all the other quantities in the following equation are real.

$$
\begin{aligned}
& \left(\frac{\partial y_{N}(x, \lambda)}{\partial x}\right)^{-1 / 2}\left(y_{N}(x, \lambda)\right)^{-1 / 4} \\
& \quad=(p(x))^{-1 / 4}\left(1+\sum_{k=1}^{N} \sum_{l=-k}^{N} q_{k l}^{\#} \lambda^{-2 k}+O\left(\lambda^{-\varepsilon N / 4}\right)\right) .
\end{aligned}
$$

Finally, let us consider $\hat{h}_{k l}$. We have that

$$
\begin{aligned}
& \left(1+\sum_{s=1}^{M} c_{s} \lambda^{-s} x^{-3 s / 2}\left(\sum_{k=0}^{N} \sum_{l=-k}^{N} h_{k l}^{s} x^{l} \lambda^{-2 k}+O\left(\lambda^{-\varepsilon N / 5}\right)\right)\right) \\
& =\left(1+\sum_{k=1}^{N} \sum_{l=-3 k}^{N} \hat{h}_{k l} x^{l / 2} \lambda^{-k}+O\left(\lambda^{-\varepsilon N / 6}\right)\right)
\end{aligned}
$$

where $h_{k l}^{s}$ is real, and $c_{s}$ has the alternating parity property on the index $s$. This is a consequence of the recurrence relation one finds upon substituting the asymptotic form of the Airey function

$$
A(t)=\operatorname{Re}\left(\frac{e^{ \pm i \pi / 4} e^{2 i t^{3 / 2} / 3}}{t^{1 / 4}}\left(1+\sum_{s=1}^{\infty} c_{s} t^{-(3 / 2) s}\right)\right)
$$

into the Airey equation

$$
\frac{d^{2}}{d y^{2}} A(y, \lambda)+\lambda^{2} y A(y, \lambda)=0
$$

Collecting the even and odd powers of $\lambda$ on the left-hand side of (11) shows that $\hat{h}_{k l}$ has the alternating parity property on the index $k$.

Putting what we know about $h_{k l}^{\#}, q_{k l}^{\#}$ and $\hat{h}_{k l}$ into $(7)$ reveals that coefficients of even powers of $\lambda$ must involve products with even total numbers of $h_{k l}^{\#}$ 's and $\hat{h}_{k l}$ 's. Note also that the $q_{k l}^{\#}$ are always accompanied by even powers of $\lambda$. Therefore the coefficients of even powers of $\lambda$ 
410 A. Ho

on the left-hand side of (7) are real. On the other hand the coefficients of odd powers of $\lambda$ are purely imaginary. Hence $w_{k}$ has the alternating parity property on the index $k$.

Acknowledgement. The author wishes to thank Charles Fefferman for many helpful discussions.

\section{References.}

[FS] Fefferman, C., Seco, L., Eigenvalues and Eigenfunctions of Ordinary Differential Operators Advances in Math. 95 (1992), 145-305.

Recibido: 28 de mayo de 1.996

Alan Ho

Department of Mathematics Princeton University Princeton, New Jersey 08544 U.S.A. alanho@math. Princeton. EDU 